\title{
Majorana fermion induced nonlocal current correlations in spin-orbit coupled superconducting wires
}

\author{
Jie Liu, ${ }^{1,}{ }^{*}$ Fu-Chun Zhang, ${ }^{1}$ and K. T. Law ${ }^{2, \dagger}$ \\ ${ }^{1}$ Department of Physics, and Center of Theoretical and Computational Physics, The University of Hong Kong, Hong Kong, China \\ ${ }^{2}$ Department of Physics, Hong Kong University of Science and Technology, Clear Water Bay, Hong Kong, China
}

(Received 24 December 2012; published 14 August 2013)

\begin{abstract}
Recent observation of zero bias conductance peaks in semiconductor wire/superconductor heterostructures has generated great interest, and there is a hot debate on whether the observation is associated with Majorana fermions (MFs). Here we study the local and crossed Andreev reflections of two normal leads attached to the two ends of a superconductor-semiconductor wire. We show that the MFs induced crossed Andreev reflections have significant effects on the shot noise of the device and strongly enhance the current-current correlations between the two normal leads. The measurements of shot noise and current-current correlations can be used to identify MFs.
\end{abstract}

DOI: 10.1103/PhysRevB.88.064509

PACS number(s): 74.45.+c, 03.75.Lm, 74.78.Na

\section{INTRODUCTION}

The search for Majorana fermions (MFs) in condensed matter systems has been an important topic in recent years as MFs are non-Abelian particles ${ }^{1,2}$ and have potential applications in quantum computations. ${ }^{3,4}$ Recent proposals suggest that MFs can appear as zero energy end states in superconducting wires constructed by inducing superconductivity on semiconductor wires with Rashba spin-orbit coupling through proximity effect. ${ }^{5-11}$ Remarkably, several experimental groups ${ }^{12-15}$ recently reported the observation of zero bias conductance peaks (ZBCPs) in Andreev reflection experiments by coupling a normal lead to the end of the aforementioned semiconductor/superconductor heterostructure. These ZBCPs were possibly due to the MF induced Andreev reflections. ${ }^{16,17}$ However, the origin of these ZBCPs remains a subject of debate. ${ }^{18-25}$

In this work, instead of studying MF induced local Andreev reflections, ${ }^{18-25}$ we explore the nonlocal properties of MFs. We show that shot noise, which can be used to probe the fractional charges, ${ }^{26-29}$ and their anyonic statistics ${ }^{30,31}$ in fractional quantum Hall states, can also be used to probe MFs in superconducting wires due to MF enhanced crossed Andreev reflections (CARs). ${ }^{32}$ To be specific, an experimental setup depicted in Fig. 1(a) is studied, in which two normal leads are attached to the two ends of a semiconductor wire with Rashba spin-orbit coupling, proximity induced $s$-wave superconductivity, and a magnetic field parallel to the wire. In the topological regime, MFs emerge as end states of the superconducting wire.

In the following sections we show that when MF end states from the two ends of the wire are strongly coupled, local Andreev reflection processes [as depicted in Fig. 1(b)] can be suppressed and the MF end states induce crossed Andreev reflections (CARs), in which an electron from one lead is reflected as a hole in a different lead [as depicted in Fig. 1(c)]. Therefore, each normal lead tunnels one electron with charge $e$ into the superconductor in each tunneling event instead of $2 e$ as in local Andreev reflection processes. As a result, the Fano factor of a normal lead, which is the ratio of the shot noise to the average current of the lead, is reduced from $2 e$ to $e$ in the CAR regime. Moreover, the current of the two

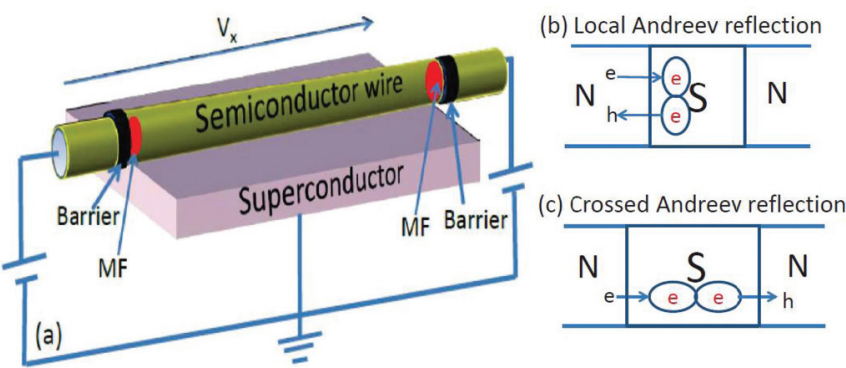

FIG. 1. (Color online) (a) A schematic setup of experiment, two normal leads are coupled to the two ends of a semiconductor wire/superconductor heterostructure which supports MFs. (b) A local Andreev reflection process. An electron from one lead is reflected as a hole in the same lead. (c) A crossed Andreev reflection process. An electron from one lead is reflected as a hole in another lead and a Cooper pair is injected into the superconductor.

spatially separated normal leads are perfectly correlated in the CAR regime since the two electrons from the two leads have to form a Cooper pair in order to tunnel into the fully gapped superconductor.

It has been pointed out that several effects such as disorder induced Andreev bounded states, ${ }^{18}$ Kondo effect, ${ }^{15,33}$ weak antilocalization, ${ }^{20}$ and reflectionless tunneling ${ }^{34,35}$ may cause ZBCPs in tunneling experiments. However, all these effects are essentially the enhancement of local Andreev reflections due to various mechanisms at the interface between a normal lead and a superconductor. These effects cannot cause perfect current-current correlations between two spatially separated leads. Therefore, the measurement of Fano factors and currentcurrent correlations can be used to identify MFs.

Moreover, an experimental setup similar to Fig. 1(a) has been fabricated recently ${ }^{14,15,36}$ and the shot noise of the set up in the topologically trivial regime is measured. ${ }^{36}$ Therefore, the measurements of the shot noise in the topologically nontrivial regime is experimentally feasible.

\section{MODEL AND FORMALISM}

To model the quasi-one-dimensional $s$-wave superconductor with Rashba spin-orbit coupling as shown in Fig. 1(a), we 


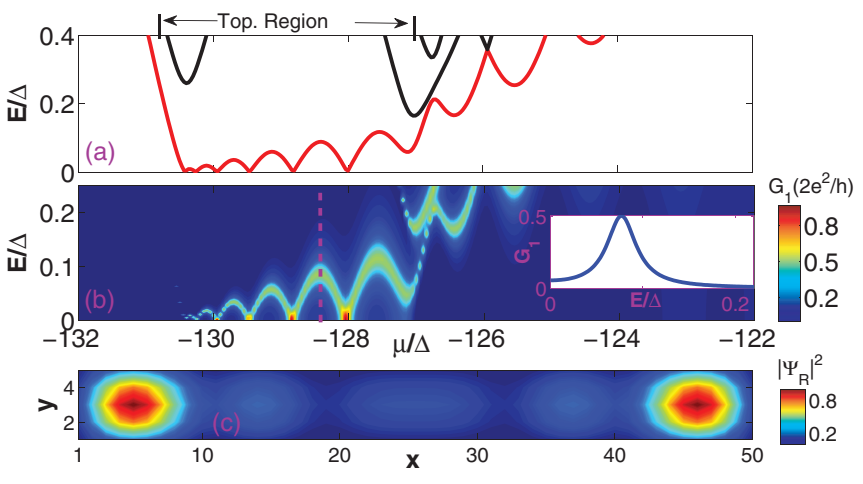

FIG. 2. (Color online) (a) The energy eigenvalues of a short quasi-1D wire versus chemical potential, the lowest energy states are indicated in red. The topological region is marked out. (b) Contour plot of differential conductance $G_{1}$ of the left lead as a function of chemical potential and electron incident energy $E$. The inset shows $G_{1}$ versus incident energy at a fixed chemical potential denoted by the vertical dashed line in (b). The height of the peak at $E=E_{M}$ is $\frac{2 e^{2}}{h} \frac{t_{L}^{2}}{t_{L}^{2}+t_{R}^{2}}$. (c) The ground state wave function $\left|\Psi_{R}\right|^{2}$ in the topological regime, with chemical potential denoted by the dashed line in (b), projected onto the $x-y$ plane. The fermionic ground state is formed by two MF end states.

use the following tight-binding model ${ }^{11,18}$ with $N_{x}, N_{y}$, and $N_{z}$ sites in the $x, y$, and $z$ directions, respectively:

$$
\begin{aligned}
H_{q 1 \mathrm{D}}= & \sum_{\mathbf{R}, \mathbf{d}, \alpha}-t\left(\psi_{\mathbf{R}+\mathbf{d}, \alpha}^{\dagger} \psi_{R, \alpha}+\text { H.c. }\right)-\mu \psi_{\mathbf{R}, \alpha}^{\dagger} \psi_{\mathbf{R}, \alpha} \\
& +\sum_{\mathbf{R}, \mathbf{d}, \alpha, \beta}-i U_{R} \psi_{\mathbf{R}+\mathbf{d}, \alpha}^{\dagger} \hat{z} \cdot(\vec{\sigma} \times \mathbf{d})_{\alpha \beta} \psi_{\mathbf{R}, \beta} \\
& +\sum_{\mathbf{R}, \alpha, \beta} \psi_{\mathbf{R}, \alpha}^{\dagger}\left[\left(V_{x} \sigma_{x}\right)_{\alpha \beta}+V_{\mathrm{imp}}(\mathbf{R}) \delta_{\alpha \beta}\right] \psi_{\mathbf{R}, \beta} \\
& +\sum_{\mathbf{R}, \alpha} \Delta \psi_{\mathbf{R}, \alpha}^{\dagger} \psi_{\mathbf{R},-\alpha}^{\dagger}+\text { H.c. }
\end{aligned}
$$

Here $\mathbf{R}$ denotes the lattice sites, and $\mathbf{d}$ denotes the three unit vectors $\mathbf{d}_{\mathbf{x}}, \mathbf{d}_{\mathbf{y}}$, and $\mathbf{d}_{\mathbf{z}}$ which connect the nearest neighbor sites in the $x, y$, and $z$ directions, respectively. The spin indexes denoted by $\alpha, \beta, t$ denotes the hopping amplitude, $\mu$ is the chemical potential, $U_{R}$ is the Rashba coupling strength, and $V_{x}$ is the Zeeman energy caused by a magnetic field along the wire in the $x$ direction. The pairing amplitude is denoted as $\Delta$ and $V_{\text {imp }}(\mathbf{R})$ is the on-site random impurity which is Gaussian distributed with variance $\overline{V_{\text {imp }}(\mathbf{R}) V_{\text {imp }}\left(\mathbf{R}^{\prime}\right)}=\omega^{2} \delta_{\mathbf{R}, \mathbf{R}^{\prime}}$. In this work, we set $V_{x}=2 \Delta$ such that the superconducting wire can support MF end states by tuning the chemical potential.

The parameters in the tight-binding model are chosen to match the corresponding values in a recent experiment as done in Ref. 18. Here $\Delta=250 \mu \mathrm{eV}, t=25 \Delta$, and $U_{R}=2.5 \Delta$. The dimensions of the wire are $N_{x} a \approx 1 \mu \mathrm{m}, N_{y} a \approx 100 \mathrm{~nm}$, and $N_{z} a \approx 60 \mathrm{~nm}$. The length of the wire is about twice the superconducting coherence length $\xi_{0} \approx t a / \Delta$ and about half the length of the experimental value in Ref. 12 . Due to the short length of the wire, as shown in Fig. 2(a), the energy of the in-gap states versus the chemical potential exhibits oscillatory behavior in the topologically nontrivial regime as the two MF end states can couple to each other and the coupling strength is an oscillating function of the chemical potential. ${ }^{24,37}$ However, as shown in Fig. 2(c), the ground state is a nonlocal fermion formed by two MF end states.

To study the current-current correlation mediated by the MF end states, two semi-infinite normal leads are attached to the two ends of the superconductor as shown in Fig. 1(a). The two normal leads are described by Eq. (1) by setting $\Delta$ to zero. The tunneling barriers are simulated by the reduced hopping amplitudes $t_{L}=t_{R}=0.4 t$ between the leads and the superconductor where $t_{L}\left(t_{R}\right)$ denotes the hopping amplitude from the left (right) lead to the superconductor. However, $t_{L / R}$ controls the width of the conductance peak and it is chosen that the width of the conductance peak is about $0.05 \Delta$ as shown in the inset of Fig. 2(b), which is larger than thermal broadening width $k_{B} T \approx 0.02 \Delta$ such that the conductance peak cannot be washed out by finite temperature effects.

We use the recursive Green's function method to calculate the scattering matrix of the model ${ }^{38}$ where the scattering matrix is related to the Green's functions of the superconducting wire by

$$
S_{i j}^{\alpha \beta}=-\delta_{i, j} \delta_{\alpha, \beta}+i\left[\Gamma_{i}^{\alpha}\right]^{1 / 2} * G^{r} *\left[\Gamma_{j}^{\beta}\right]^{1 / 2} .
$$

Here $S_{i j}^{\alpha, \beta}$ is an element of the scattering matrix which denotes the scattering amplitude of a $\beta$ particle from lead $j$ to an $\alpha$ particle in lead $i$, where $i, j=1$ or 2 . 1 and 2 denote the left and the right lead, respectively. The electron $(e)$ or hole (h) channels are denoted by $\alpha, \beta, \in\{e, h\} . G^{r}$ is the retarded Green's function of the superconducting wire. $\Gamma_{i}^{\alpha}=i\left[\left(\Sigma_{i}^{\alpha}\right)^{r}-\right.$ $\left.\left(\Sigma_{i}^{\alpha}\right)^{a}\right]$, where $\left(\Sigma_{i}^{\alpha}\right)^{r(a)}$ is the $\alpha$ particle retarded (advanced) self-energy of lead $i$.

With the scattering matrix, the average current $\bar{I}_{i}$ of lead $i$, the differential shot noise $P_{i j}$ and shot noise $C_{i j}$ can be calculated as 39,40

$$
\begin{aligned}
\bar{I}_{i} & =\frac{\mathrm{e}}{h} \int_{0}^{\mathrm{eV}} \sum_{j, \alpha} \operatorname{Tr}\left[I-\operatorname{sgn}(\alpha) S_{i j}^{e \alpha}(E)^{\dagger} S_{i j}^{e \alpha}(E)\right] d E, \\
P_{i j}(E) & =\frac{2 \mathrm{e}^{2}}{h} \sum_{\alpha, k \beta \neq l \beta^{\prime}} \operatorname{sgn}(\alpha) \operatorname{Tr}\left[S_{i l}^{e \beta^{\prime} \dagger} S_{i k}^{e \beta} S_{j k}^{\alpha \beta \dagger} S_{j l}^{\alpha \beta^{\prime}}(E)\right], \\
C_{i j} & =\int_{0}^{e V} P_{i j}(E) d E,
\end{aligned}
$$

where $\operatorname{sgn}(\alpha)=1$ if $\alpha=e$ and $\operatorname{sgn}(\alpha)=-1$ if $\alpha=h$. In this work we set the chemical potential of the two normal leads to be the same and the voltage bias between the leads and the superconductor to be $V$. Physically, $C_{i j}=\int_{-\infty}^{+\infty} \overline{\delta I_{i}(0) \delta I_{j}(t)} d t$ measures the current fluctuation of leads $i$ and $j$, where $\delta I_{i}=$ $I_{i}(t)-\bar{I}_{i}$ denotes the deviation of the current at time $t$ with respect to the average current $\bar{I}_{i}$. At low temperatures with $k_{B} T \ll e V$, the current fluctuation is dominated by the shot noise ${ }^{41}$ and $C_{i j}$ is reduced to the shot noise. On the other hand, $\frac{d C_{i j}}{d V}=e P_{i j}$ is the differential shot noise caused by electrons with incident energy $E$. In this work, the current and noise are calculated at zero temperature.

\section{CURRENT}

In this section we focus on the tunneling current near the topological regime where only one transverse subband 
is occupied and there are MF end states. Due to the oscillatory nature of the MF wave functions, the coupling strength of the MFs oscillate and the resulting coupling energy oscillates as $E_{M} \approx \frac{\hbar^{2} k_{F}}{m^{*} \xi_{0}} e^{-2 N_{x} a / \xi_{0}} \cos \left(k_{F} N_{x}\right)$, where $k_{F}$ is the Fermi momentum which is a function of chemical potential and magnetic field and $m^{*}$ is the effective band mass. ${ }^{37}$ The energy spectrum of the superconducting wire is shown in Fig. 2(a). The topological regime is marked out in Fig. 2(a). As the chemical potential increases and a second transverse subband is occupied, the superconductor becomes topologically trivial. $^{3,11}$

To study the MF end states, we calculate the differential conductance $d \bar{I}_{1} / d V$ using Eq. (3). The contour plot of the differential conductance of the left lead $G_{1}=d \bar{I}_{1} / d V$ as a function of electron incident energy $E=e V$ and the chemical potential is shown in Fig. 2(b). As expected, the MFs manifest themselves by inducing conductance peaks. However, in the presence of the second lead, the height of the conductance peak is reduced to $G_{1}\left(E_{M}\right)=d \bar{I}_{1} /\left.d V\right|_{e V=E_{M}} \approx \frac{2 e^{2}}{h} \frac{t_{L}^{2}}{t_{L}^{2}+t_{R}^{2}}$ as shown in the inset of Fig. 2(b). The numerical calculations in Fig. 2(b) correspond to the case with $t_{L}=t_{R}$. As a result, $G_{1}\left(E_{M}\right)=0.5 \frac{2 e^{2}}{h}$.

Another interesting point for tunneling into a superconductor with two strongly coupled MFs is that at low incident energy $E \ll E_{M}$, the differential conductance $G_{1}(E) \propto$ $\frac{2 e^{2}}{h} \frac{t_{L}^{2} t_{R}^{2}}{E_{M}^{2}}$ depends on the product of $t_{L}$ and $t_{R}$. This means that an electron from a normal lead cannot tunnel into the superconductor unless a second normal lead is present. This is a manifestation of the fact that local Andreev reflection processes are suppressed and the current is purely caused by CAR processes.

\section{DIFFERENTIAL SHOT NOISE}

To probe the MF induced CAR processes, we note that the local and CAR processes can be distinguished experimentally by measuring the Fano factors ${ }^{41}$ of the normal leads. The Fano factor is the ratio of the shot noise to the average current. Physically the Fano factor measures the electric charge leaving a lead at each tunneling event given that the tunneling amplitude is small.

In this section we first study the energy dependence of the Fano factors for electrons with incident energy $E$. The contour plot of the Fano factor $F(E)=P_{11}(E) / G_{1}(E)$ is shown in Fig. 3(a). Here $P_{11}(E)$ is the differential shot noise $\frac{1}{e} \frac{d C_{i i}}{d V}$. Here $P_{11}(E)$ is caused by electrons with incident energy $E$ from the left normal lead and $G_{1}(E)$ is the differential conductance of the left normal lead. Similarly, the crossed current-current correlator $P_{12}(E)$, normalized by the average differential conductance $\bar{G}(E)=\frac{1}{2}\left(G_{1}+G_{2}\right)$, is shown in Fig. 3(b). $P_{12}(E)$ measures the current-current correlations between the left and the right leads. Even though $P_{i j}$ are more difficult to measure experimentally than $C_{i j}$, they give detailed information about different tunneling processes as a function of $E$ as shown below.

From Figs. 3(a) and 3(e) it is evident that the Fano factor $F(E)=P_{11}(E) / G_{1}(E)$ at $E=0$ is the electron charge $e$ in the topological regime. Similarly, it can be shown that the
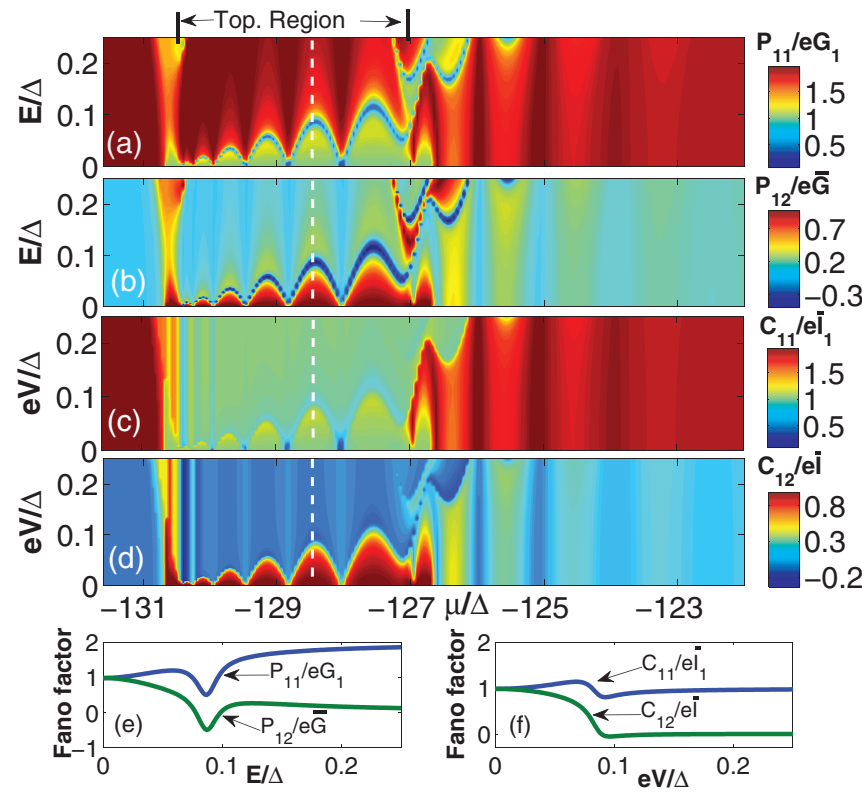

FIG. 3. (Color online) (a) Contour plot of Fano factor $P_{11} / e G_{1}$ for electrons with incident energy $E$ at chemical potential $\mu$. (b) Contour plot of $P_{12} / e \bar{G}$. (c) Contour plot of $C_{11} / e \bar{I}_{1}$ as a function of voltage bias $e V$ and chemical potential. (d) Contour plot of $C_{12} / e \bar{I}$. (e) The $P_{11} / e G_{1}$ (blue line) and $P_{12} / e \bar{G}$ (green line) as a function of incident energy $E$ at a fixed chemical potential denoted by the dashed lines in (a) and (b), respectively. (f) The $C_{11} / e \bar{I}_{1}$ (blue line) and $C_{12} / e \bar{I}$ (red line) as a function of voltage bias at fixed chemical potential denoted by the dashed lines in (c) and (d).

Fano factor for the right lead is $P_{22}(E=0) / G_{2}(E=0)=e$. This indicates that for each tunneling event, each normal lead contributes one electron in the tunneling process. Moreover, it is evident from Figs. 3(b) and 3(e) that $P_{12}(E=0) / \bar{G}=e$. As pointed out in Ref. 32, the cross correlator $P_{12}$ is bound by the relation $2\left|P_{12}\right| \leqslant P_{11}+P_{22}$ for any stochastic process. At $E=0$ we have $2\left|P_{12}(E)\right|=P_{11}(E)+P_{22}(E)$. This indicates that the two leads are perfectly correlated with each other such that a Cooper pair is injected into the superconductor at each tunneling event. It is important to note that the almost perfect current-current correlation persists as long as $E \lesssim E_{M}$. This is in sharp contrast to the topologically trivial regime as shown in Fig. 3(a) in which local Andreev reflection processes dominate and the Fano factor for each lead is $2 e$. The tunneling currents of the two leads are only weakly correlated in the absence of MFs as shown in Fig. 3(b).

\section{SHOT NOISE}

In this section we study the shot noise $C_{i j}$, which is the integration of the differential shot noise over the incident energy as defined in Eq. (3). The contour plots of $C_{11}$ and $C_{12}$, normalized by $\bar{I}_{1}$ and $\bar{I}=\frac{1}{2}\left(\bar{I}_{1}+\bar{I}_{2}\right)$, respectively, as a function of chemical potential and voltage bias, are shown in Figs. 3(c) and 3(d). The Fano factor $C_{i i} / \bar{I}_{i}$ gives the charge leaving lead $i$ at each tunneling event. As expected, in the CAR regime with $E \lesssim E_{M}, C_{i i} / \bar{I}_{i} \approx e$. In this case, the two leads are almost perfectly correlated as $C_{12} / \bar{I} \approx e$ as shown in Fig. 3(f). 


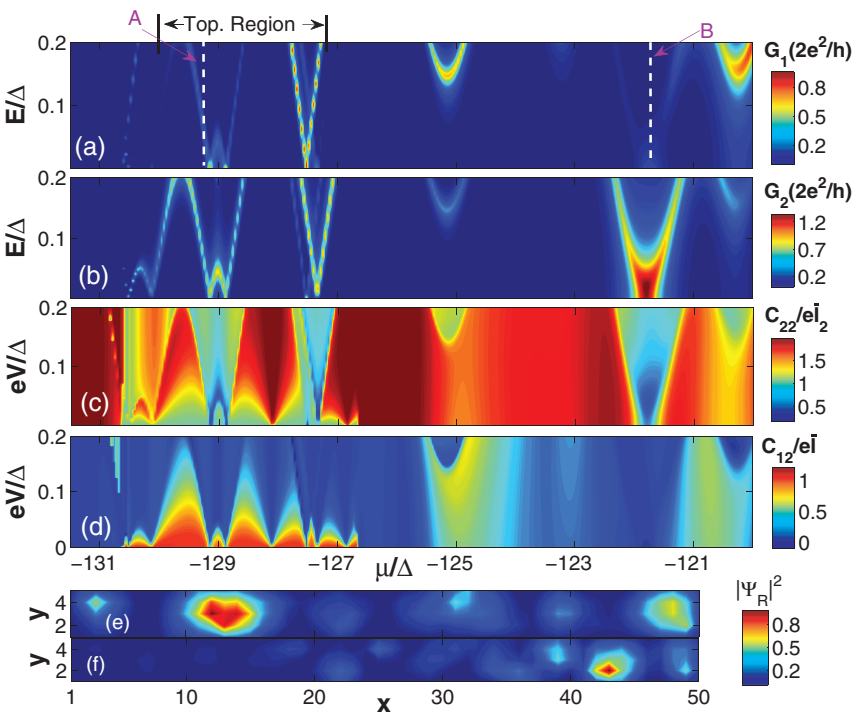

FIG. 4. (Color online) Random disorder with $\omega=16 \Delta$ is present for all the figures. (a) and (b) The contour plots of the conductance $G_{1}$ and $G_{2}$, respectively. (c) and (d) The Fano factors $C_{22} / e \bar{I}_{2}$ and $C_{12} / e \bar{I}$, respectively. (e) The ground state wave function $\left|\Psi_{\mathbf{R}}\right|^{2}$ in the topological regime with $\mu=-129.4 \Delta$ [indicated by dashed line A in (a)]. The dimensions of the wire are $N_{x}=50 a$ and $N_{y}=5 a$. (f) The ground state wave function in the trivial regime with $\mu=-120.8 \Delta$ [indicated by dashed line B in (a)].

Outside of the topological regime, $C_{i i} / \bar{I}_{i} \approx 2 e$ as local Andreev reflection processes dominate. Moreover, the cross correlation between the two leads $C_{12}$ is significant only when the incident energy of electrons satisfies $E \ll E_{M}$ in the topological regime as shown in Fig. 3(d).

\section{DISORDER EFFECT}

The observation of the ZBCPs in recent tunneling experiments is an important step in the search for MFs. ${ }^{12-15}$ However, as argued in Ref. 18, fermionic end states formed by two MF end states in the topologically trivial regime can also induce ZBCPs in the presence of disorder. Therefore, it is important to distinguish the fermionic end states from the true MF end states. In this section we show that the shot noise caused by a localized fermionic end state and the shot noise caused by two spatially separated MF end states are different.

To study the disorder effect, ${ }^{42}$ on-site random disorder with Gaussian distribution with $\omega=16 \Delta$ is added to the superconducting wire. The disorder strength are chosen such that nearly zero energy Andreev bound states can be induced in the topologically trivial regime. ${ }^{18}$ The contour plots of the differential conductance $G_{1}$ and $G_{2}$ for the left and right leads are shown in Figs. 4(a) and 4(b), respectively. It is important to note that in the topologically trivial regime where two transverse subbands of the wire are occupied, a fermionic end state which has energy close to zero is induced by disorder at $\mu \approx-122 \Delta$. The ground state wave function at $\mu=-121.8 \Delta$ (projected onto the $x-y$ plane) is shown in Fig. 4(f) and it is evident that the ground state is localized at the right end of the wire. As expected, this zero energy fermionic end state induces a strong conductance peak for the right normal lead as shown in Fig. 4(b). Therefore, it is difficult to distinguish this fermionic state from a true MF end state by measuring the conductance alone.

However, since the fermionic end state at $\mu \approx-122 \Delta$ is a localized state, the cross current-current correlation $C_{12} / e \bar{I}$ induced by this state is small as shown in Fig. 4(d). On the contrary, $C_{12} / e \bar{I}$ is close to 1 in the topological regime at $E \ll E_{M}$ as shown in Fig. 4(d).

To further identify the topological regime, we note that the Fano factor of the right lead $C_{22} / e \bar{I}_{1}$ at $E \approx 0$ is close to 1 only in the topological regime as shown in Fig. 4(c). The ground state wave function in the topological regime at $\mu=-129.2 \Delta$ is shown in Fig. 4(e). It is evident that this fermionic end state, which can mediate CARs, is a nonlocal fermionic state and its wave function has significant distribution at both ends of the wire. Therefore, the experimental signatures of $C_{12} / e \bar{I} \approx 1$ and $C_{11} / e \bar{I}_{1} \approx 1$ at $E \approx 0$ can be used to distinguish MFs from local fermions.

\section{CONCLUSION}

We show that the MF induced CARs change the shot noise and strongly enhance the cross current-current correlations between two leads. The measurements of the Fano factor $e$ of the leads and the strong current-current correlation at small voltage bias can be used to detect MFs. The effects of different magnetic field strength and sample size as well as the transport properties in the multisubband regime are discussed in the Appendix. It is shown that the MF enhanced CAR effect discussed in this work is very robust and independent of the details of the parameters used.

\section{ACKNOWLEDGMENTS}

We thank H. Barranger, D. Feldman, H. Jiang, P. A. Lee, X. J. Liu, A. C. Potter, Q. F. Sun, and especially M. Heiblum for insightful discussions. K.T.L. and J.L. thank the support of HKRGC through Grant 605512 and HKUST3/CRF09. F.C.Z. thanks the support of RGC HKU707211 and AOE/P-04/08.

\section{APPENDIX}

\section{Effective Hamiltonian}

When a single transverse subband of the superconducting wire is occupied, the wire is in the topological regime with two Majorana end states, we expect the transport properties of a N/TS/N junction at $e V \ll \Delta$ can be qualitatively described by the effective Hamiltonian $H_{\text {eff }}=H_{L}+H_{M}+H_{T}$, where

$$
\begin{aligned}
H_{N} & =-i v_{f} \sum_{\alpha \in L / R} \int_{-\infty}^{+\infty} \psi_{\alpha}^{\dagger}(x) \partial_{x} \psi_{\alpha}(x) d x \\
H_{M} & =i E_{M} \gamma_{L} \gamma_{R} \\
H_{T} & =-i\left\{\tilde{t}_{L} \gamma_{L}\left[\psi_{L}^{\dagger}(0)+\psi_{L}(0)\right]+\tilde{t}_{R} \gamma_{R}\left[\psi_{R}^{\dagger}(0)+\psi_{R}(0)\right]\right\} .
\end{aligned}
$$

Here $H_{N}$ is the Hamiltonian of the left and right normal leads and $\psi_{L / R}$ denotes a fermion operator of the left (right) normal lead. $v_{f}$ is the corresponding Fermi velocity of the leads. $H_{M}$ describes the two coupled Majorana fermions, where $E_{M}$ is the coupling strength between the two MF end states $\gamma_{L}$ and $\gamma_{R}$. The coupling between the leads and the MFs are described 
by $H_{T}$, where the coupling strengths are denoted by $\tilde{t}_{L}$ and $\tilde{t}_{R}$, respectively.

This model was first introduced in Refs. 32 and 43 and the scattering matrix of the Hamiltonian can be found easily using the equation of motion approach. ${ }^{16}$ We denote the incoming states of the electrons and holes with momentum $k$ in the left and right leads by $\psi_{L / R k}(-)$ and $\psi_{L / R-k}^{\dagger}(-)$, respectively. The electron and hole scattering states are denoted by $\psi_{L / R k}(+)$ and $\psi_{L / R-k}^{\dagger}(+)$, respectively. The scattering matrix $S$ is defined as

$$
\left(\begin{array}{c}
\psi_{L k}(+) \\
\psi_{R k}(+) \\
\psi_{L-k}^{\dagger}(+) \\
\psi_{R-k}^{\dagger}(+)
\end{array}\right)=\left(\begin{array}{cc}
S^{e e} & S^{e h} \\
S^{h e} & S^{h h}
\end{array}\right)\left(\begin{array}{c}
\psi_{L k}(-) \\
\psi_{R k}(-) \\
\psi_{L-k}^{\dagger}(-) \\
\psi_{R-k}^{\dagger}(-)
\end{array}\right) .
$$

Using the equation of motion method ${ }^{16}$ and following the notations in Ref. 32, we have

$$
S(E) \equiv\left(\begin{array}{ll}
S^{e e} & S^{e h} \\
S^{h e} & S^{h h}
\end{array}\right)=\left(\begin{array}{cc}
1+A & A \\
A & 1+A
\end{array}\right),
$$

where

$$
A=Z^{-1}\left(\begin{array}{cc}
-i\left(E+i \frac{2 \tilde{t}_{R}^{2}}{v_{f}}\right) \frac{2 \tilde{t}_{L}^{2}}{v_{f}} & \frac{-2 E_{M} \tilde{t}_{L} \tilde{t}_{R}}{v_{f}} \\
\frac{2 E_{M} \tilde{t}_{L} \tilde{t}_{R}}{v_{f}} & -i\left(E+i \frac{2 \tilde{t}_{L}^{2}}{v_{f}}\right) \frac{2 \tilde{t}_{R}^{2}}{v_{f}}
\end{array}\right) .
$$

and $Z=E_{M}^{2}-\left(E+i 2 \tilde{t}_{R}^{2} / v_{f}\right)\left(E+i 2 \tilde{t}_{L}^{2} / v_{f}\right)$. From the scattering matrix, the local Andreev reflection amplitude for the, say, left lead is $-i\left(E+i \frac{2 \tilde{t}_{R}^{2}}{v_{f}}\right) \frac{2 \tilde{t}_{L}^{2}}{v_{f}} / Z$. When the two Majorana fermions are not coupled and at zero voltage bias with $E_{M}=0$ and $E=0$, the local Andreev reflection amplitude is 1. This is called resonant Andreev reflections in Ref. 16. However, when the two Majorana fermions are strongly coupled with $E_{M} \gg E$ and $4 \tilde{t}_{L}^{2} \tilde{t}_{R}^{2} / v_{f}^{2}$, the local Andreev reflection is strongly suppressed. It is interesting to note that when $|E|=\left|E_{M}\right| \gg\left|\tilde{t}_{L} \tilde{t}_{R} / v_{f}\right|$, the local Andreev reflection amplitude is $t_{L}^{2} /\left(t_{L}^{2}+t_{R}^{2}\right)$ and results in a conductance peak of $\frac{2 e^{2}}{h} \tilde{t}_{L}^{2} /\left(\tilde{t}_{L}^{2}+\tilde{t}_{R}^{2}\right)$. All these simple analytic results match the numerical results in the corresponding regime very well, as shown in Fig. 2(b) of the main text. For example, in the main text, the effective coupling between the leads to the superconductor are set to be equal such that $\tilde{t}_{L}=\tilde{t}_{R}$ and the effective Hamiltonian predicts a conductance peak of $0.5 * 2 e^{2} / h$ at $E=E_{M}$. This is verified in the inset of Fig. 2(b).

From the scattering matrix it is evident that the crossed Andreev reflection amplitude is $\frac{2 E_{M} \tilde{t}_{L} \tilde{t}_{R}}{v_{f}} / Z$. Therefore, the crossed Andreev reflection is zero if the two Majorana fermions are not coupled when $E_{M}=0$. As shown above, when $E_{M} \gg E$ and $4 \tilde{t}_{L}^{2} \tilde{t}_{R}^{2} / v_{f}^{2}$, the local Andreev reflection is strongly suppressed to the order of $E \tilde{t}_{L}^{2} / v_{f} E_{M}^{2}$. However, in this regime, the crossed Andreev reflection is of order $\frac{2 \tilde{t}_{L} \tilde{t}_{R}}{E_{M}}$. This results in a conductance of order $\frac{2 e^{2}}{h} \frac{\tilde{L}_{L}^{2} \tilde{t}_{R}^{2}}{E_{M}^{2}}$ at zero voltage bias $E=0$. Moreover, in this regime, it can be shown using the scattering matrix and Eq. (3) of the main text that $P_{11} /\left.\bar{G}_{1}\right|_{E \ll E_{M}} \approx e$, as shown by the numerical results. It can also be verified that at $E=0,2\left|P_{12}(E)\right|=P_{11}(E)+P_{22}(E)$ such that the two normal leads are perfectly correlated to each

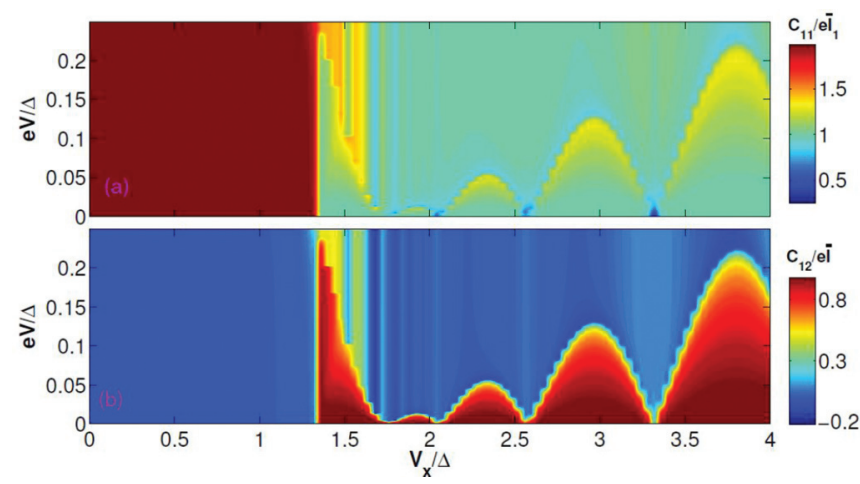

FIG. 5. (Color online) (a) Contour plot of $C_{11} / e \bar{I}_{1}$ as a function of voltage bias and magnetic field $V_{x}$. (b) Contour plot of $C_{12} / e \bar{I}$.

other. This is also consistent with the numerical simulations as shown in Fig. 3.

It is interesting to note that the elastic cotunneling amplitudes from the left lead to the right lead equal the crossed Andreev reflection amplitude. However, since the left lead and the right lead have equal chemical potential and electrons can also tunnel from the right lead to the left lead, there is no net current from the left lead to the right lead and vice versa. Therefore, elastic cotunneling processes do not contribute to the net current of the normal leads.

\section{Current-current correlations as a function of magnetic field}

It is important to note that for the short wire geometry, $E_{M}$ oscillates as a functions of magnetic field and chemical potential. ${ }^{24,37}$ The energy eigenvalues as a function of the magnetic field strength along the wire are shown in Fig. 5. The chemical potential is $\mu=-130 \Delta$. It is evident that when $V_{x}$ is smaller than the superconducting gap, there are no in-gap states. When $V_{x}$ is larger than the bulk pairing gap, the superconducting wire is tuned to the topological regime with Majorana end states. Due to the short wire geometry, the coupling energy oscillates as a function of magnetic field. As expected, in when $E_{M} \gg e V$, crossed Andreev reflection processes dominate.

\section{Current-current correlations when multiple subbands are occupied}

It is important to note that in realistic experiments it is possible that multitransverse subbands of the superconducting wire are occupied. ${ }^{12,15}$ In the topological regime with an odd number of transverse subbands occupied, and if the number of occupied subbands is larger than one, the appearance of Majorana end states is accompanied by the appearance of finite energy fermionic end states. ${ }^{11}$ Therefore, it is important to show that the measurement of the shot noise and currentcurrent correlations can be used to probe the topological regime even in the presence of other fermionic end states. In this section we first identify the topological regime by plotting the energy eigenstates of a superconducting wire. When the chemical potential is near the band bottom, only one or two transverse subbands are occupied as shown in the main text. As the chemical potential increases, more transverse subbands of the superconducting wire are occupied. When three subbands 

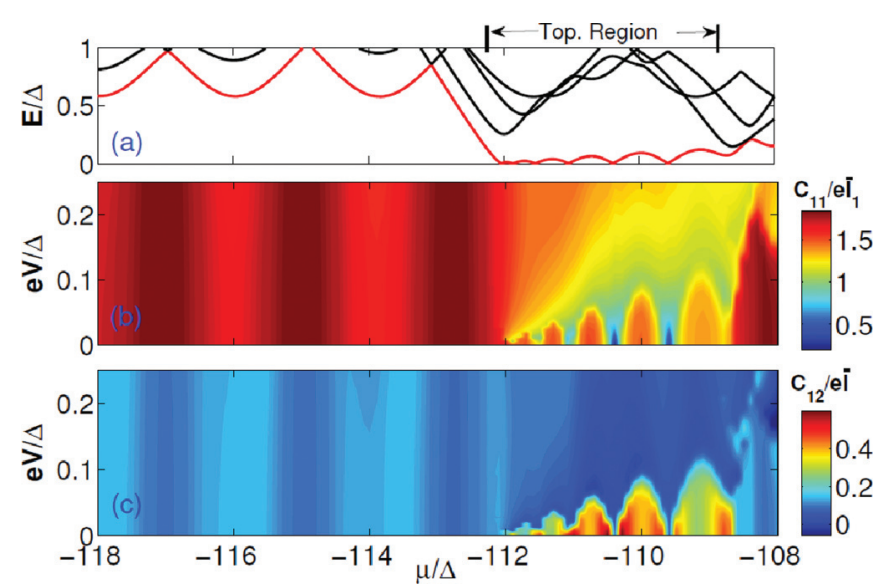

FIG. 6. (Color online) (a) The energy eigenvalues as a function of chemical potential. The topological regime with Majorana fermion end states are marked out. (b) Contour plot of $C_{11} / e \bar{I}_{1}$ as a function of voltage bias and chemical potential. (c) Contour plot of $C_{12} / e \bar{I}$.

are occupied, the wire is again in the topological regime. The energy eigenvalues as a function of chemical potential in the regime where three transverse subbands are occupied are shown in Fig. 6(a).

The Fano factor of the left lead $C_{11} / e \bar{I}_{1}$ and the currentcurrent correlations of the two normal leads $C_{12} / e \bar{I}$ are shown in Figs. 6(a) and 6(c), respectively. It is evident that in the topological regime with $e V \lesssim E_{M}$, we have $C_{11} / e \bar{I}_{1} \approx 1$ and $C_{12} / e \bar{I} \approx 1$. Therefore, the Fano factors and current-current correlations can still be used to probe the topological regime even in the multisubband cases.

\section{The effect of the sample length}

It is shown in the main text that the enhanced crossed Andreev reflection regimes occur when the coupling energy between the Majorana fermions is larger than the voltage bias such that $E_{M} \gg e V$. Moreover, the voltage bias has to be larger than the temperature scale $e V \gg k_{B} T$ such that the shot noise can dominate the thermal noise. Fortunately these conditions can be easily satisfied in the semiconductor/superconductor heterostructure. As shown in Figs. 2 and 3 of the main text, the maximum coupling energy can be in the order of $0.1 \Delta$, which is much larger than $k_{B} T$. Assuming that $\Delta=0.25$ $\mathrm{meV}$ as shown experimentally and $T=20 \mathrm{mK}$, we have $k_{B} T \approx 0.01 \Delta$.

Since the coupling strength of the two Majorana end states has the form $E_{M} \approx \frac{\hbar^{2} k_{F}}{m^{*} \xi_{0}} e^{-2 N_{x} a / \xi_{0}} \cos \left(k_{F} N_{x}\right)$, the maximum $E_{M}$ decreases exponentially as a function of the distance between the Majorana end states. The localization length is the superconducting coherence length $\xi_{0} \approx t a / \Delta \approx 25 a$. In the main text, a wire length of $N_{x} a=50 a$ is assumed. In this section, results using wires with lengths $N_{x} a=40 a$ and $N_{x} a=70 a$ are presented. As expected, the maximum $E_{M}$ is increased when the wire is shortened. When $N_{x} a=40 a$, the maximum $E_{M} \approx 0.15 \Delta$. When $N_{x} a=70 a$, the maximum $E_{M}$ is reduced to about $0.05 \Delta$ and the crossed Andreev reflection regime is more difficult to observe. Nevertheless, the length of the wire should not be much shorter than the superconducting coherence length. Otherwise, the current-current correlations

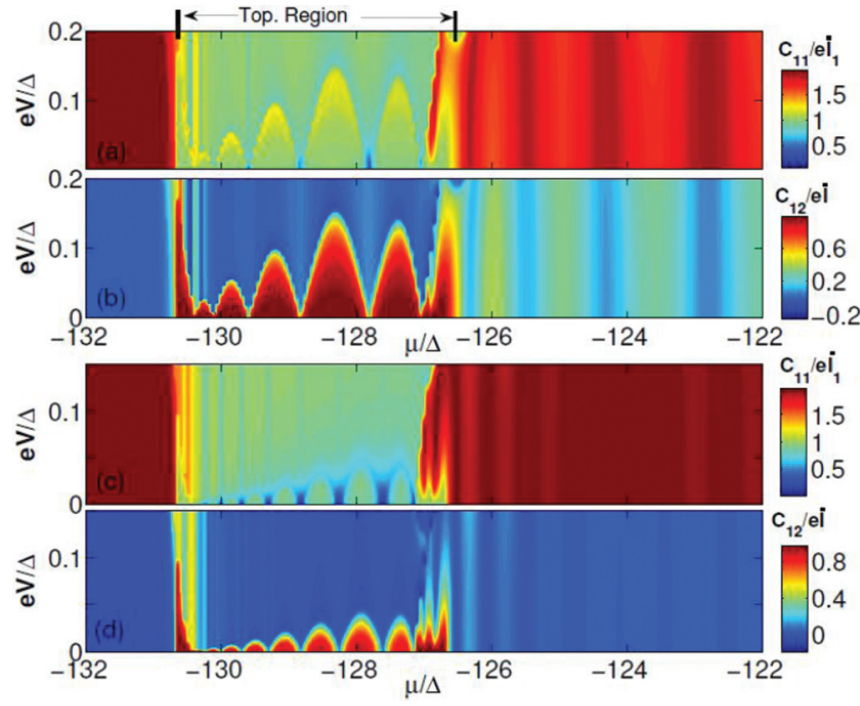

FIG. 7. (Color online) $N_{x}=40$ for (a) and (b). $N_{x}=70$ for (c) and (d). (a) and (c) Contour plots of $C_{11} / e \bar{I}_{1}$ as a function of voltage bias $e V$ and chemical potential. (b) and (d) Contour plots of $C_{12} / e \bar{I}$.

between the two leads can be significant even in the absence of Majorana fermions. For example, it is evident from Figs. 7(b) and $7(\mathrm{~d})$ that $C_{12} / \bar{I}$ in the topologically trivial regime of the shorter wire with $N_{x} a=40 a$ is much larger than $C_{12} / \bar{I}$ in the topologically trivial regime of the wire with $N_{x} a=70 a$.

\section{Discussion on mechanisms which can enhance local Andreev reflections}

As mentioned in the main text, several effects such as disorder induced Andreev bounded states, ${ }^{18}$ Kondo effect, ${ }^{15,33}$ weak antilocalization, ${ }^{20}$ and reflectionless tunneling ${ }^{34,35}$ may cause zero bias conductance peaks in tunneling experiments. However, all these effects are essentially the enhancement of local Andreev reflections due to various mechanisms at the interface between a normal lead and a superconductor. In this section we argue that these effects cannot enhance crossed Andreev reflections.

First, reflectionless tunneling is due to the disorder induced constructive interference between time-reversal invariant scattering paths at the normal lead. The constructive interferences enhance the local Andreev reflection amplitude. This enhancement happens at a local section of the normal lead. Therefore, they cannot enhance crossed Andreev reflection processes.

Second, weak antilocalization effect at a N-S junction is another candidate for inducing zero bias conductance peaks. Weak antilocalization at a N-S junction is caused by the destructive interference effect between multiple scattering paths of electrons and holes near the N-S interface. In the weak antilocalization regime, the local Andreev reflection amplitude is increased causing an increased ZBCP as shown in Ref. 20. Since only local Andreev reflection processes are involved, there is no enhancement of nonlocal correlations between leads.

Third, ordinary fermionic Andreev bound states caused by nonmagnetic disorder or magnetic disorder, localized at 
the end of a superconducting wire, can induce conductance peaks. If the Andreev bound states have nearly zero energy, the conductance peak appears at near zero bias. It is shown in the main text that these disorder induced Andreev bound states cannot induce crossed Andreev reflections, even though they can induce almost resonant local Andreev reflections.

Finally, Kondo effect can also give rise to zero bias conductance peaks. Kondo effect is the enhancement of the effective coupling between two leads induced by a magnetic impurity as the temperature is lower than the Kondo temperature. Kondo effect is usually suppressed if one of the leads becomes superconducting. However, in the presence of a magnetic field, the proximity induced pairing gap $\Delta$ can be reduced. In the regime where $\Delta$ is smaller than the Kondo temperature $k_{B} T_{\text {Kondo }}$, a conductance peak can emerge.

However, similar to other effects discussed above, Kondo effects are local processes induced by a local magnetic impurity/quantum dot, which cannot enhance the current-current correlations between two spatially separated leads. *jliuphy@hku.hk

†phlaw@ust.hk

${ }^{1}$ D. A. Ivanov, Phys. Rev. Lett. 86, 268 (2001).

${ }^{2}$ J. Alicea, Y. Oreg, G. Refael, F. von Oppen, and M. P. A. Fisher, Nat. Phys. 7, 412 (2011).

${ }^{3}$ A. Kitaev, arXiv:cond-mat/0010440.

${ }^{4}$ C. Nayak, S. H. Simon, A. Stern, M. Freedman, and S. Das Sarma, Rev. Mod. Phys. 80, 1083 (2008).

${ }^{5}$ J. D. Sau, R. M. Lutchyn, S. Tewari, and S. Das Sarma, Phys. Rev. Lett. 104, 040502 (2010).

${ }^{6}$ S. Fujimoto, Phys. Rev. B 77, 220501(R) (2008).

${ }^{7}$ M. Sato, Y. Takahashi, and S. Fujimoto, Phys. Rev. B 82, 134521 (2010).

${ }^{8}$ J. Alicea, Phys. Rev. B 81, 125318 (2010).

${ }^{9}$ R. M. Lutchyn, J. D. Sau, and S. Das Sarma, Phys. Rev. Lett. 105, 077001 (2010).

${ }^{10}$ Y. Oreg, G. Refael, and F. von Oppen, Phys. Rev. Lett. 105, 177002 (2010).

${ }^{11}$ A. C. Potter and P. A. Lee, Phys. Rev. B 83, 094525 (2011).

${ }^{12}$ V. Mourik, K. Zuo, S. M. Frolov, S. R. Plissard, E. P. A. M. Bakkers, and L. P. Kouwenhoven, Science 336, 1003 (2012).

${ }^{13}$ M. T. Deng, C. L. Yu, G. Y. Huang, M. Larsson, P. Caroff, and H. Q. Xu, Nano Lett. 12, 6414 (2012).

${ }^{14}$ A. Das, Y. Ronen, Y. Most, Y. Oreg, M. Heiblum, and H. Shtrikman, Nat. Phys. 8, 887 (2012).

${ }^{15}$ H. O. H. Churchill, V. Fatemi, K. Grove-Rasmussen, M. T. Deng, P. Caroff, H. Q. Xu, and C. M. Marcus, Phys. Rev. B 87, 241401(R) (2013).

${ }^{16}$ K. T. Law, P. A. Lee, and T. K. Ng, Phys. Rev. Lett. 103, 237001 (2009).

${ }^{17}$ M. Wimmer, A. R. Akhmerov, J. P. Dahlhaus, and C. W. J. Beenakker, New J. Phys. 13, 053016 (2011).

${ }^{18}$ J. Liu, A. C. Potter, K. T. Law, and P. A. Lee, Phys. Rev. Lett. 109, 267002 (2012).

${ }^{19}$ D. Bagrets and A. Altland, Phys. Rev. Lett. 109, 227005 (2012).

${ }^{20}$ D. I. Pikulin, J. P. Dahlhaus, M. Wimmer, H. Schomerus, and C. W. J. Beenakker, New J. Phys. 14, 125011 (2012).

${ }^{21}$ G. Kells, D. Meidan, and P. W. Brouwer, Phys. Rev. B 85, 060507(R) (2012).
${ }^{22}$ S. Tewari, T. D. Stanescu, J. D. Sau, and S. Das Sarma, Phys. Rev. B 86, 024504 (2012).

${ }^{23}$ F. Pientka, G. Kells, A. Romito, P. W. Brouwer, and F. von Oppen, Phys. Rev. Lett. 109, 227006 (2012).

${ }^{24}$ D. Rainis, L. Trifunovic, J. Klinovaja, and D. Loss, Phys. Rev. B 87, 024515 (2013).

${ }^{25}$ E. Prada, P. San-Jose, and R. Aguado, Phys. Rev. B 86, 180503(R) (2012).

${ }^{26}$ L. Saminadayar, D. C. Glattli, Y. Jin, and B. Etienne, Phys. Rev. Lett. 79, 2526 (1997).

${ }^{27}$ R. de Picciotto, M. Reznikov, M. Heiblum, V. Umanski, G. Bunin, and D. Mahalu, Nature (London) 389, 162 (1997).

${ }^{28}$ M. Reznikov, R. de Picciotto, T. G. Griffiths, M. Heiblum, and V. Umansky, Nature (London) 399, 238 (1999).

${ }^{29}$ M. Dolev, M. Heiblum, V. Umansky, A. Stern, and D. Mahalu, Nature (London) 452, 829 (2008).

${ }^{30}$ D. E. Feldman, Y. Gefen, A. Kitaev, K. T. Law, and A. Stern, Phys. Rev. B 76, 085333 (2007).

${ }^{31}$ K. T. Law, Phys. Rev. B 77, 205310 (2008).

${ }^{32}$ J. Nilsson, A. R. Akhmerov, and C. W. J. Beenakker, Phys. Rev. Lett. 101, 120403 (2008).

${ }^{33}$ E. J. H. Lee, X. Jiang, R. Aguado, G. Katsaros, C. M. Lieber, and S. De Franceschi, Phys. Rev. Lett. 109, 186802 (2012).

${ }^{34}$ B. J. van Wees, P. de Vries, P. Magnee, and T. M. Klapwijk, Phys. Rev. Lett. 69, 510 (1992).

${ }^{35}$ I. K. Marmorkos, C. W. J. Beenakker, and R. A. Jalabert, Phys. Rev. B 48, 2811 (1993).

${ }^{36}$ A. Das, Y. Ronen, M. Heiblum, D. Mahalu, A. V. Kretinin, and H. Shtrikman, Nat. Commun. 3, 1165 (2012).

${ }^{37}$ S. Das Sarma, J. D. Sau, and T. D. Stanescu, Phys. Rev. B 86, 220506 (2012).

${ }^{38}$ P. A. Lee and D. S. Fisher, Phys. Rev. Lett. 47, 882 (1981); D. S. Fisher and P. A. Lee, Phys. Rev. B 23, 6851 (1981).

${ }^{39}$ Q. F. Sun and X. C. Xie, J. Phys.: Condens. Matter 21, 344204 (2009).

${ }^{40}$ M. P. Anantram and S. Datta, Phys. Rev. B 53, 16390 (1996).

${ }^{41}$ Ya. M. Blanter and M. Buttiker, Phys. Rep. 366, 1 (2000).

${ }^{42}$ P. W. Brouwer, M. Duckheim, A. Romito, and F. von Oppen, Phys. Rev. B 84, 144526 (2011).

${ }^{43}$ C. J. Bolech and E. Demler, Phys. Rev. Lett. 98, 237002 (2007). 